\title{
Conhecimento dos profissionais da saúde sobre o autocuidado em hanseníase
}

\author{
Knowledge of health professionals about self-care in leprosy \\ Conocimiento de profesionales de la salud sobre el cuidado personal en lepra
}

Recebido: 05/10/2021 | Revisado: 16/10/2021 | Aceito: 19/10/2021 | Publicado: 21/10/2021

\author{
Débora Ferreira Angelim \\ ORCID: https://orcid.org/0000-0002-6589-897X \\ Centro Universitário Vale do Salgado, Brasil \\ E-mail: deboraferreira001@gmail.com \\ Rafael Bezerra Duarte \\ ORCID: https://orcid.org/0000-0002-2280-0864 \\ Centro Universitário Vale do Salgado, Brasil \\ E-mail: rafaelduarte@univs.edu.br \\ Marcos Raí da Silva Tavares \\ ORCID: https://orcid.org/0000-0002-5899-9641 \\ Centro Universitário Vale do Salgado, Brasil \\ E-mail: marcosrai@univs.edu.br \\ Jeynna Suyanne Pereira Venceslau \\ ORCID: https://orcid.org/0000-0002-7853-4251 \\ Centro Universitário Vale do Salgado, Brasil \\ E-mail: jeynnasuyanne@univs.edu.br
}

\begin{abstract}
Resumo
O estudo tem como objetivo, avaliar como se apresenta o conhecimento prévio dos profissionais de saúde acerca do autocuidado em hanseníase nas Estratégias de Saúde da Família do município de Icó-CE. Trata-se de um estudo de campo, exploratório de abordagem quantitativa e qualitativa que foi realizado durante os meses de janeiro e fevereiro de 2021 na cidade de Icó, localizada na região Centro-Sul do estado do Ceará. A amostra foi constituída por 11 profissionais da saúde que acompanham pacientes com Hanseníase de oito Estratégias de Saúde da Família da cidade de Icó-CE, houve predominância do sexo feminino (11,1\% homens e 88,8 \% mulheres). Destaca-se que 88,8\% dos entrevistados já tinham algum curso ou conhecimento sobre a hanseníase. Os dados obtidos com este estudo, assemelham-se à literatura, revelando aspectos educacionais e práticos falhos acerca da hanseníase, o que permitiram conhecer o nível de conhecimento dos profissionais de saúde sobre a doença, bem como entender sobre as repercussões que podem ser ocasionados nos pacientes com hanseníase devido a falha da gestão em saúde no processo de educação permanente e atualizações. Reconhecer estas limitações e ter estratégias para transformá-las a favor de uma formação contínua de educação em saúde e atualizações entre os membros da equipe interprofissional são desafios para que se torne possível fazer avanços acerca das práticas do autocuidado e autonomia do paciente hansênico em relação ao tratamento e à doença.
\end{abstract}

Palavras-chave: Hanseníase; Profissionais da saúde; Autocuidado; Sistema Único de Saúde.

\begin{abstract}
The study aims to evaluate how the prior knowledge of health professionals about self-care in leprosy in the Family Health Strategies in the city of Icó-CE is presented. This is an exploratory field study with a quantitative and qualitative approach that was carried out during the months of January and February 2021 in the city of Icó, located in the Center-South region of the state of Ceará. The sample consisted of 11 health professionals who accompany patients with leprosy from eight Family Health Strategies in the city of Icó-CE, with a predominance of females ( $11.1 \%$ men and $88.8 \%$ women). It is noteworthy that $88.8 \%$ of respondents already had some course or knowledge about leprosy. The data obtained from this study are similar to the literature, revealing flawed educational and practical aspects about leprosy, which allowed to know the level of knowledge of health professionals about the disease, as well as to understand the repercussions that can be caused in the leprosy patients due to failure of health management in the process of continuing education and updates. Recognizing these limitations and having strategies to transform them in favor of continuous training in health education and updates among members of the interprofessional team are challenges to make it possible to make advances in the self-care practices and autonomy of the leprosy patient in relation to the treatment and disease.
\end{abstract}

Keywords: Leprosy; Health care professionals; Self care; Unified Health System.

\section{Resumen}

El estudio tiene como objetivo evaluar cómo se presenta el conocimiento previo de los profesionales de la salud sobre el autocuidado en lepra en las Estrategias de Salud de la Familia en la ciudad de Icó-CE. Se trata de un estudio de 
campo exploratorio con enfoque cuantitativo y cualitativo que se llevó a cabo durante los meses de enero y febrero de 2021 en la ciudad de Icó, ubicada en la región Centro-Sur del estado de Ceará. La muestra estuvo conformada por 11 profesionales de la salud que acompañan a pacientes con lepra de ocho Estrategias de Salud de la Familia en la ciudad de Icó-CE, con predominio del sexo femenino (11,1\% hombres y 88,8\% mujeres). Es de destacar que el 88,8\% de los encuestados ya tenía algún curso o conocimiento sobre la lepra. Los datos obtenidos de este estudio son similares a la literatura, revelando aspectos pedagógicos y prácticos deficientes sobre la lepra, que permitieron conocer el nivel de conocimiento de los profesionales de la salud sobre la enfermedad, así como comprender las repercusiones que se pueden ocasionar en la lepra. pacientes por fallas en la gestión sanitaria en el proceso de formación continua y actualización. Reconocer estas limitaciones y contar con estrategias para transformarlas a favor de la formación continua en educación para la salud y actualización entre los miembros del equipo interprofesional son desafíos para hacer posible avanzar en las prácticas de autocuidado y autonomía del enfermo de lepra en relación con la tratamiento y enfermedad.

Palabras clave: Lepra; Profesionales de la salud; Autocuidado; Sistema Único de Salud.

\section{Introdução}

A hanseníase é uma doença crônica de origem infectocontagiosa causada pelo vírus Mycobacterium Leprae, também conhecido como bacilo de Hansen, que ocasiona modificações imunológicas, levando a manifestação de alterações neurológicas, cutâneas, oculares e viscerais. Acomete principalmente os nervos periféricos tanto nos membros superiores como inferiores, podendo manifestar-se de forma sistêmica (Morais \& Furtado, 2018).

O comprometimento dos nervos periféricos promove a perda gradativa de sensibilidade cutânea e alterações motoras, podendo evoluir para atrofias, paresias, paralisias musculares e incapacidades físicas, que podem levar ao desenvolvimento de deformidades, gerando limitações para o indivíduo acometido e a uma redução nas atividades de vida diária (Moura et al, 2017).

O grau de incapacidade física pode variar de acordo com a fase em que se encontra a doença podendo ser crônica ou aguda. Os indivíduos que se encontram na fase aguda da doença possuem um melhor prognóstico se realizarem o tratamento com acompanhamento dos serviços de saúde da atenção básica. Já na fase crônica o tratamento se torna mais limitado e os comprometimentos causados ficam cada vez mais difíceis de serem revertidos apresentando sequelas em níveis mais severos (Rodrigues et al, 2015).

A prática do autocuidado apresenta-se como uma estratégia fundamental no tratamento da hanseníase, uma vez que consiste em técnicas e procedimentos que buscam prevenir as incapacidades físicas ou evitar o agravamento dos comprometimentos ocasionados por esta patologia. Além disso, a prática de autocuidado pode ser realizada em domicílio e o conhecimento acerca da doença e das estratégias de tratamento é um direito do indivíduo acometido pela hanseníase (Lima et al, 2018).

A Estratégia de Saúde da Família (ESF) ressalta as práticas de promoção da saúde e prevenção de doenças, buscando uma solução para os problemas mais comuns da população. Logo, a hanseníase vem sendo cada vez mais identificada como um problema de saúde pública e caracterizada como um dos principais desafios para os profissionais de saúde devido a sua elevada prevalência e pelo impacto negativo causado na população (Rodrigues et al, 2015).

O nível de informação dos profissionais sobre os fatores gerais e específicos da hanseníase e da prática do autocuidado deve ser satisfatório para que haja qualidade na assistência e no serviço de atendimento ao indivíduo com diagnóstico da doença, visando promover uma melhora nas deformidades e incapacidades físicas e consequentemente melhorando a qualidade de vida do indivíduo (Oliveira et al, 2017).

A hanseníase, caracteriza-se como uma das doenças mais negligenciadas, a qual apresenta um alto índice de prevalência na cidade de Icó-CE, tendo manifestados 64 casos novos no ano de 2017 de acordo com o boletim epidemiológico de hanseníase do estado do Ceará (Brasil, 2018). Além disso, é considerada como um grave problema de saúde pública no Brasil e em países em desenvolvimento. Tal situação pode ser evidenciada pela escassez de informações científicas acerca da 
hanseníase e da prática do autocuidado.

Dessa forma, o conhecimento dos profissionais da saúde acerca do autocuidado em hanseníase se torna indispensável e de grande relevância, visto que as incapacidades físicas podem ser evitadas ou reduzidas. Portanto, este estudo contribuirá na avaliação e análise das informações que os profissionais possuem acerca do autocuidado em hanseníase.

Diante da importância de os profissionais de saúde possuírem os conhecimentos adequados sobre hanseníase e autocuidado, visto que esta patologia é tratada a nível da Atenção Básica de Saúde (ABS), foi levantado a seguinte problemática: Como se apresenta o conhecimento prévio dos profissionais de saúde acerca do autocuidado em hanseníase? O estudo tem como objetivo avaliar como se apresenta o conhecimento prévio dos profissionais de saúde acerca do autocuidado em hanseníase através da investigação de como é definida a hanseníase, quais as suas principais sequelas e repercussões na qualidade de vida do paciente e quais os tipos de técnicas de autocuidado são orientados.

\section{Metodologia}

Este estudo compõe o projeto de pesquisa "Conhecimento dos profissionais da saúde sobre o autocuidado em hanseníase", aprovado pelo Comitê de Ética em Pesquisa da Faculdade Leão Sampaio e aprovado com número CAAE: 38093220.0.0000.5048. Os sujeitos do estudo tiveram acesso ao Termo de Consentimento Livre e Esclarecido (TCLE), que foi incluído como a primeira página do formulário, onde só poderiam responder ao questionário após concordarem com o TCLE e marcarem como assinado, expressando seu desejo de participar da pesquisa, atendendo aos princípios éticos, conforme a Resolução n. 466/2012 do Conselho Nacional de Saúde.

Trata-se de um estudo de campo, exploratório de abordagem quali-quantitativa. O estudo de campo é caracterizado pelo levantamento de dados, no ambiente onde os fatos ocorrem (Ludwig, 2012). A pesquisa quantitativa caracteriza-se pela apresentação de amostras de caráter amplo por meio de dados estatísticos e a qualitativa se trata de uma pesquisa que analisa e interpreta aspectos descrevendo de forma detalhada acerca das informações obtidas (Marconi \& Lakatos, 2010). A pesquisa exploratória tem como finalidade oferecer informações acerca de um objeto de estudo e nortear a formulação de hipóteses (Gil, 2014).

A pesquisa foi realizada durante os meses de janeiro e fevereiro de 2021, nas Estratégia de Saúde da Família da Zona Urbana da cidade de Icó, localizada na região Centro-Sul do estado do Ceará. As Estratégias de Saúde da Família (ESF) da Zona Urbana são: Posto de Saúde São Vicente de Paula, Programa Saúde Da Família do Centro, Programa Saúde da Família Conjunto Cidade Nova I e II, Programa Saúde Da Família de São Geraldo, Posto De Saúde Alto Manoel Mariano I e II, Programa Saúde da Família Conjunto Gama.

Foram convidados a participar da pesquisa todos os profissionais da saúde (médicos, enfermeiros, técnicos de enfermagem e agentes comunitária da saúde) que trabalham nas ESF da zona urbana do município de Icó, compondo uma equipe de 34 profissionais da saúde. A execução da pesquisa se deu através de uma amostra não probabilística por conveniência, totalizando 11 sujeitos. Um primeiro contato foi realizado com 18 profissionais, onde 04 não aceitaram participar da pesquisa, 02 estavam de férias e 01 não utiliza meios tecnológicos.

Como critérios de inclusão, foram elencados: profissionais de ambos os sexos que possuam formação em alguma área da saúde, que atuem no acompanhamento com pacientes hansênicos nas ESF da Zona Urbana e aceitem participar da pesquisa, assinando o Termo de Consentimento Pós-esclarecido (TCPE). Como critério de exclusão, os profissionais de saúde que não preencherem completamente e corretamente o questionário de coleta de dados, bem como os que estavam de licença maternidade, licença médica ou férias regulamentares.

A pesquisa foi realizada através da aplicação de um questionário online semiestruturado, elaborado pelos pesquisadores, utilizando a plataforma Google Forms. Ao responderem ao questionário os participantes especificaram seu 
nível de conhecimento acerca do autocuidado em hanseníase. Dessa forma, o roteiro contém 6 questões subjetivas direcionadas ao autocuidado e 4 objetivas elaboradas com base no modelo de perguntas binárias e de múltipla escolha, que proporcionam informações específicas sobre o objetivo da pesquisa. A utilização das questões discursivas objetiva melhor abranger o pensamento e opinião dos participantes.

Os participantes do estudo foram recrutados através do contato com o coordenador(a) de todos as ESF que viabilizou o compartilhamento do link do questionário aos profissionais da saúde para execução da pesquisa. É importante ressaltar, que o questionário foi aplicado individualmente, onde o participante respondeu no seu tempo e quando teve disponibilidade.

O procedimento analítico se constitui em dois tópicos: o primeiro se constitui na descrição das informações por meio de estatística descritiva (análise gráfica). Para operacionalização dos dados foi utilizado o software Statistical Package for Social Sciences (SPSS), sendo apresentados os principais pontos a serem discutidos de acordo com a literatura científica de referência sobre hanseníase. O segundo tópico foi retratado o aspecto qualitativo do estudo, onde as falas foram transcritas na íntegra e iniciou-se a análise dos discursos obtidos utilizando-se a estratégia metodológica do Discurso do Sujeito Coletivo (DSC), que consiste em realizar uma síntese do conteúdo discursivo.

\section{Resultados e Discussão}

A amostra foi constituída por 11 profissionais da saúde atuantes nas estratégias de Saúde da Família que acompanham pacientes com Hanseníase no município de Icó. Quanto a formação destes profissionais, participaram da pesquisa 6 enfermeiras, 1 médico, 1 técnica de enfermagem e 3 Agentes Comunitária de Saúde (ACS). Entre os entrevistados, houve predominância do sexo feminino (9,09\% homens e 90,9 \% mulheres) e a maior parte da amostra apresenta alguma especialização, sendo 45,4\% com pós-graduação latu sensu, 9,09\% stricto sensu e 45,4\% não apresenta nenhum tipo de especialização. Além disso, dentre os 8 ESF da zona urbana da cidade de Icó, apenas 1 ESF não entrou na pesquisa devido a não viabilização do contato com os profissionais (Tabela1). 
Tabela 1 - Perfil dos participantes da pesquisa.

\begin{tabular}{|c|c|c|c|c|c|c|}
\hline Participante & Formação & Especialização & Idade & & ESF & $\begin{array}{l}\text { Tempo de } \\
\text { trabalho }\end{array}$ \\
\hline 1 & Médico & $\begin{array}{l}\text { Especialista em Medicina } \\
\text { da Família }\end{array}$ & 35 & & $\begin{array}{l}\text { Cidade nova I e } \\
\text { II }\end{array}$ & 6 anos \\
\hline 2 & ACS & - & 58 & & $\begin{array}{l}\text { Cidade nova I e } \\
\text { II }\end{array}$ & 28 anos \\
\hline 3 & Enfermeira & - & 37 & & $\begin{array}{l}\text { Cidade nova I e } \\
\text { II }\end{array}$ & 1 ano \\
\hline 4 & Enfermeira & $\begin{array}{l}\text { Especialista em Saúde da } \\
\text { Família. Gestão da Clínica. } \\
\text { Saúde da Criança e do } \\
\text { Adolescente }\end{array}$ & 42 & & $\begin{array}{l}\text { São Vicente de } \\
\text { Paula }\end{array}$ & 18 anos \\
\hline 5 & Enfermeira & $\begin{array}{l}\text { Especialista em } \\
\text { Enfermagem do Trabalho }\end{array}$ & 29 & & $\begin{array}{l}\text { Alto Manoel } \\
\text { Mariano I e II }\end{array}$ & $\begin{array}{l}5 \text { anos e } 6 \\
\text { meses }\end{array}$ \\
\hline 6 & Enfermeira & $\begin{array}{l}\text { Especialista em Saúde da } \\
\text { Família }\end{array}$ & 52 & & $\begin{array}{l}\text { Alto Manoel } \\
\text { Mariano I e II }\end{array}$ & 20 anos \\
\hline 7 & ACS & - & 42 & & $\begin{array}{l}\text { Alto Manoel } \\
\text { Mariano I e II }\end{array}$ & 23 anos \\
\hline 8 & ACS & - & 36 & & $\begin{array}{l}\text { Alto Manoel } \\
\text { Mariano I e II }\end{array}$ & 12 anos \\
\hline 9 & Enfermeira & Especialista em UTI adulto & 33 & & Gama & 4 anos \\
\hline 10 & Enfermeira & $\begin{array}{l}\text { Mestre em Saúde da } \\
\text { Família }\end{array}$ & 49 & & São Geraldo & 15 anos \\
\hline 11 & $\begin{array}{l}\text { Técnica } \\
\text { Enfermagem }\end{array}$ & - & & 24 & São Geraldo & 1 ano \\
\hline
\end{tabular}

Fonte: Autores (2021).

Os dados obtidos com este estudo, assemelham-se à literatura, revelando aspectos educacionais e práticos falhos acerca da hanseníase, o que permitiram conhecer o nível de conhecimento dos profissionais de saúde sobre a doença, bem como entender sobre as repercussões que podem ser ocasionados nos pacientes com hanseníase devido a falha da gestão em saúde no processo de educação permanente e atualizações.

Desta forma, serão apresentadas as informações quantitativas que compõem amostras de caráter amplo e estatístico e abrangem questionamentos sobre: o conhecimento destes profissionais acerca do programa de controle de hanseníase do Ministério da Saúde; o acompanhamento de pacientes com hanseníase; importância da prática de autocuidado e a avaliação neurológica simplificada e sua utilização em pacientes com hanseníase.

No Gráfico 1 observa-se a distribuição sobre o conhecimento dos participantes acerca do programa de controle da Hanseníase proposto pelo Ministério da Saúde. 
Gráfico 1 - Você conhece o programa de controle da Hanseníase proposto pelo Ministério da Saúde?

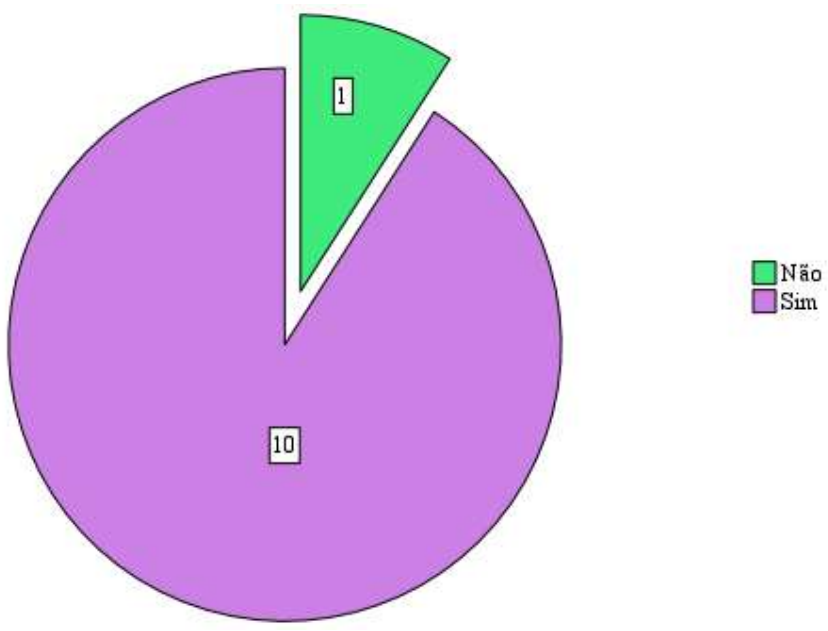

Fonte: Autores (2021).

É de extrema importância salientar que apesar de todas as falhas demonstradas no processo de educação sobre hanseníase, de 11 profissionais de saúde entrevistados, apenas 1 deles não tem conhecimento sobre o programa de controle proposto pelo Ministério da Saúde, que é baseado no diagnóstico precoce, tratamento, prevenção de incapacidades e vigilância dos contatos domiciliares.

Destaca-se que um dos pontos abordados durante a aplicação do questionário foi referente ao conhecimento dos participantes sobre a hanseníase, onde $81,8 \%$ dos entrevistados afirmaram que já tinham algum curso ou conhecimento acerca da patologia. Por conseguinte, o saber dos participantes acerca do Programa Nacional de Controle da Hanseníase (PNCH) torna-se complementar ao fato de que a maior parte da amostra apresenta um conhecimento prévio sobre hanseníase.

A Educação Permanente em Saúde (EPS), visa oferecer uma formação e qualificação dos profissionais inseridos nos serviços públicos de saúde, com o objetivo de transformar as práticas profissionais e a própria equipe multidisciplinar com base nas necessidades e dificuldades existentes no sistema para atender a população (Ferreira et al, 2019).

Em concordância, Ceretta e colaboradores (2012), afirmam que a prática de Educação em Saúde facilita o envolvimento e a interação entre profissionais de saúde, nesse sentido, Ceccim (2005), reforça que a educação em saúde deve ser disseminada por toda rede do SUS, sendo o processo educacional contínuo uma das metas da saúde coletiva.

O PNCH é composto por cinco componentes, dentre eles: vigilância epidemiológica; gestão; atenção integral desde o diagnóstico, tratamento, prevenção de incapacidades, reabilitação e inclusão social; comunicação e educação; pesquisa através de centros de referência e pesquisas operacionais (Brasil, 2009).

Considerando que todas essas ações devem ser executadas assiduamente em toda a rede de atenção primária a saúde devido ao grande potencial de ocasionar incapacidades físicas, deve-se garantir a todas as pessoas atingidas pela hanseníase, uma atenção especializada e assistência integral em todas as diferentes complexidades.

O Gráfico 2 retrata quantos profissionais da saúde das ESF's da zona urbana da cidade de Icó-CE que foram inclusos na pesquisa, realizam o acompanhamento de pacientes com hanseníase. 
Gráfico 2 - Você faz o acompanhamento de pacientes com hanseníase?

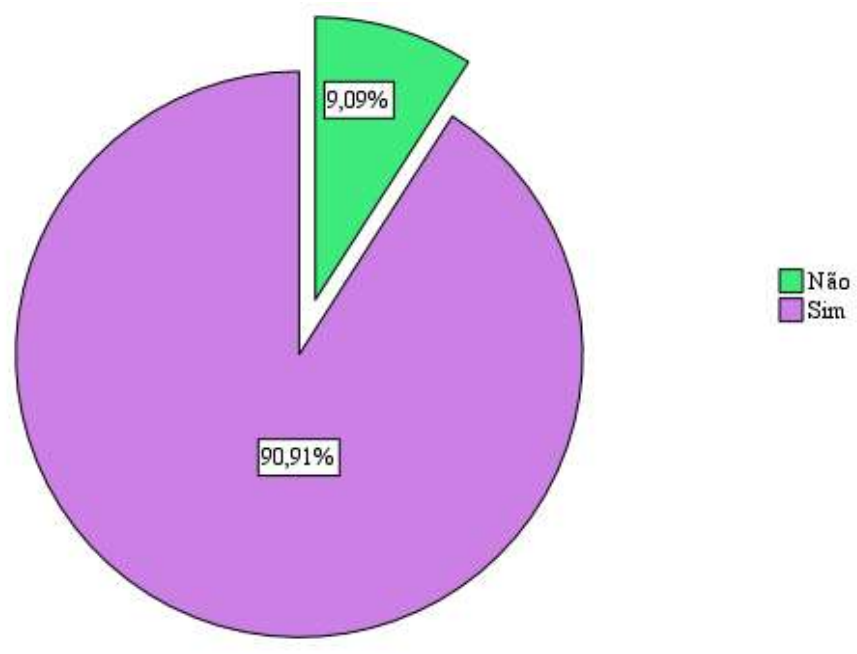

Fonte: Autores (2021).

Observa-se que 90,91\% da amostra acompanha pacientes com Hanseníase e apenas 9,09\% dos profissionais não fazem esse acompanhamento, o que não exclui o acompanhamento futuro necessitando de conhecimento prévio da patologia para proporcionar um atendimento de qualidade, e para isso, torna-se indispensável que haja continuidade da educação em saúde, englobando todos os aspectos do tratamento para portadores de hanseníase.

É de extrema importância que diversos profissionais de saúde possam avançar na garantia da universalidade do acesso e integralidade da atenção trazendo melhorias no cuidado ao usuário e para o próprio trabalho em equipe na Atenção Primária à Saúde (APS). Deste modo, a APS requer profissionais capacitados que avancem em direção ao trabalho em equipe 11 (Previato, \& Baldissera, 2018).

O profissional de saúde que atua na APS trabalha com o suporte e assistência a diversos pacientes com inúmeras patologias, entre elas, a hanseníase, e para que haja um melhor acompanhamento desses pacientes, é de extrema importância que haja capacitações e uma maior inserção de um trabalho multiprofissional.

No Gráfico 3, são apresentados a discriminação dos dados em uma escala de relevância (0 a 5), referente ao quanto estes profissionais da saúde consideram importante a prática do autocuidado, sendo 1 muito pouco e 5 extremamente importante. 
Gráfico 3 - O quanto você considera importante a prática de autocuidado?

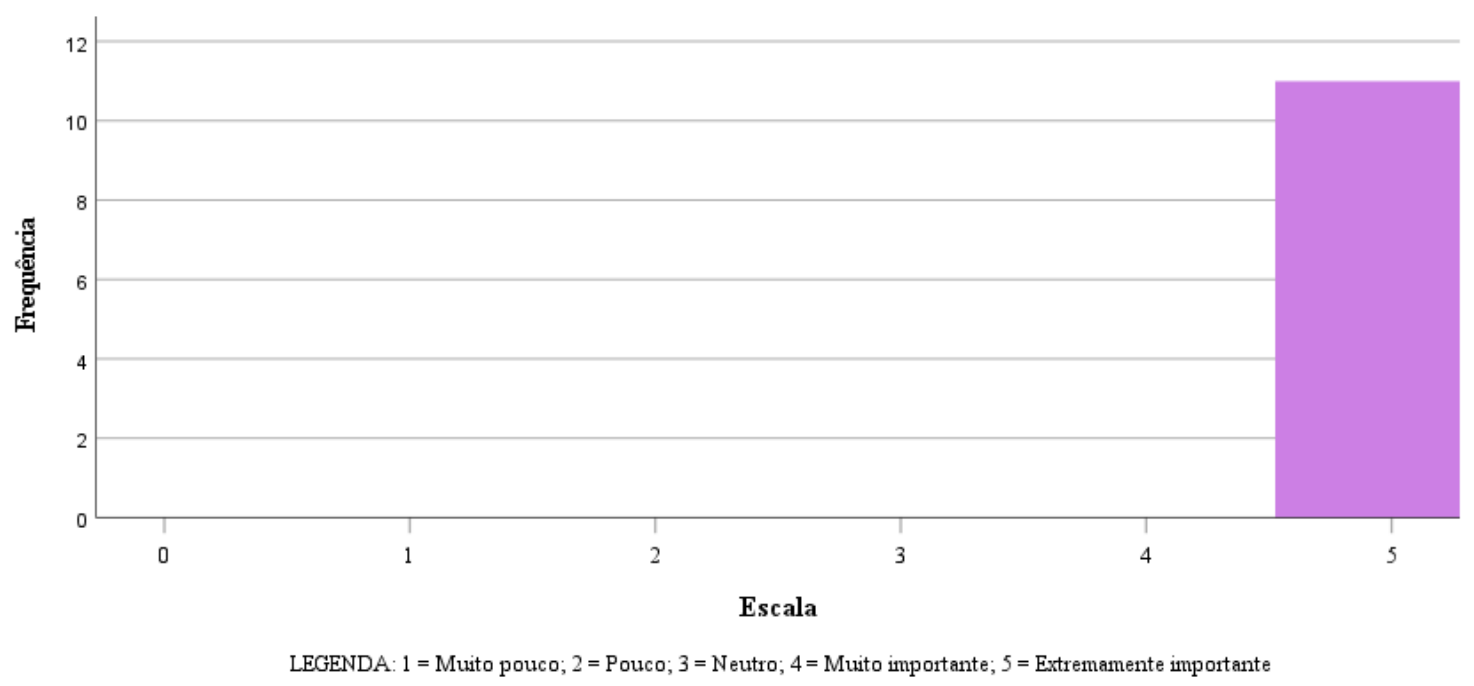

Fonte: Autores (2021)

Observa-se que $100 \%$ da amostra considera extremamente importante a prática de autocuidado em Hanseníase. Enfatiza-se que orientações sobre o autocuidado devem fazer parte da rotina do atendimento de todos os profissionais de saúde que acompanham pacientes com hanseníase. Todos os profissionais participantes afirmaram ser de extrema importância a prática de autocuidado, dessa forma, deve ser recomendado a organização de grupos de autocuidado e os pacientes devem ser estimulados a manterem continuidade dessas práticas.

Segundo Galan e colaboradores (2016), o exercício do autocuidado consiste na capacidade humana de engajar-se nas realizações das orientações adequadas para o cuidado diário e ainda é afetado por fatores condicionantes como: gênero, idade, estado de saúde, orientação sociocultural, sistema familiar, fatores socioeconômicos e ambientais, adequação e disponibilidade de recursos.

Souza e colaboradores (2014) afirmam que a interação entre o paciente com hanseníase e a equipe multiprofissional torna-se indispensável para reconhecer a necessidade da prática do autocuidado, assim como para o desenvolvimento da autonomia. A eficácia do processo de autocuidado depende exclusivamente do comprometimento do indivíduo, o que se destaca a importância de reconhecer as mudanças biopsicossociais decorrentes do processo da doença.

Grupos de Apoio ao Autocuidado em hanseníase (GAC), apresentam-se, de acordo com D’Azevedo e colaboradores (2018), como uma ferramenta indispensável na manutenção do tratamento e para a prevenção de incapacidades. Além disso, promovem uma maior interação social, troca de experiências entre seus integrantes e facilitam para os profissionais de saúde repassar as orientações de autocuidado individuais adequadas.

O Gráfico 4 retrata sobre o conhecimento e utilização da Avaliação Neurológica Simplificada pelos profissionais, no acompanhamento ao paciente com Hanseníase. 
Gráfico 4 - Avaliação Neurológica Simplificada e sua utilização na avaliação de pacientes com hanseníase.

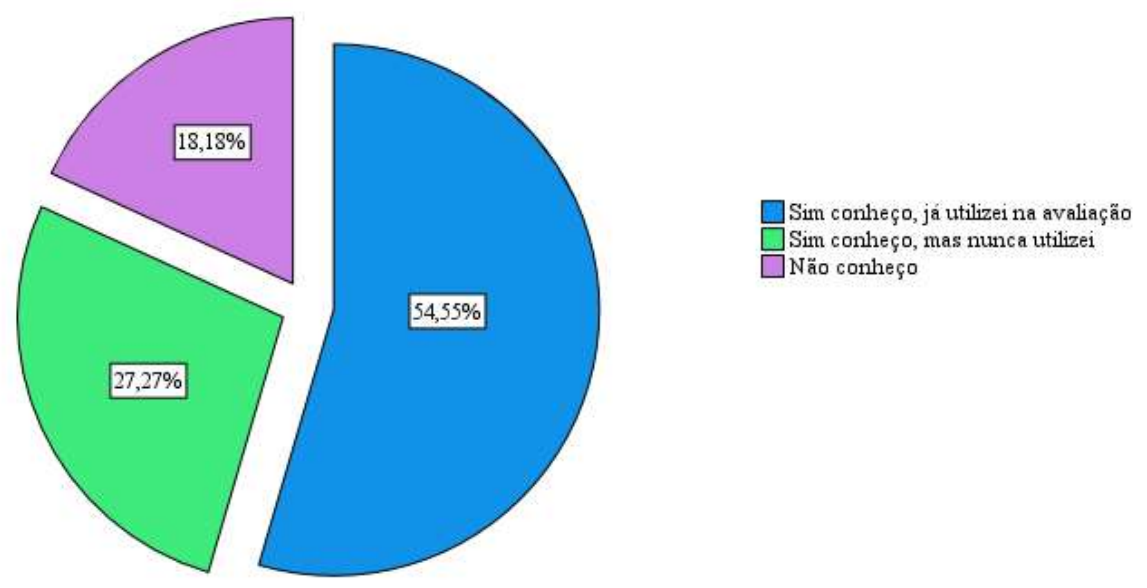

Fonte: Autores (2021)

Dentre os profissionais de saúde atuantes no acompanhamento de pacientes com hanseníase nas ESF da zona urbana da cidade de Icó, 54,55\% conhecem a avaliação neurológica simplificada e já utilizaram na avaliação, 27,27\% conhecem, mas nunca utilizaram e por fim, 18,18\% não conhecem a avaliação neurológica simplificada.

A avaliação neurológica simplificada é imprescindível para verificação da integridade da função neural, investigação de incapacidades e deformidades, e deve ser realizada com frequência para que assim possam ser tomadas todas as medidas adequadas de prevenção e tratamento (Brasil, 2019).

Para tanto, corroborando com Lehman (1997) é necessário que seja realizada de forma sistemática e regular, integrando ações de controle à hanseníase, podendo identificar precocemente danos neurais que, se tratados adequadamente, podem evitar o surgimento de deformidades e incapacidades. Além disso, oferece aos profissionais de saúde subsídios para uma melhor determinação de condutas e acompanhamento.

Ressalta-se que a avaliação neurológica simplificada é recomendada para verificar a integridade da função neural, dessa forma, apresenta-se como uma ferramenta necessária para o diagnóstico precoce. A utilização de práticas de autocuidado, o exame de contatos, conclusão do tratamento e a qualidade do serviço prestado a todas as pessoas acometidas pela hanseníase, constituem um conjunto de estratégias importantes para a redução da carga da doença (Brasil, 2020).

A análise das informações qualitativas coletadas foi realizada através do Discurso do Sujeito Coletivo, transcrevendo as respostas na íntegra e as agrupando de acordo com os objetivos da pesquisa, como podem ser observadas a seguir.

O Quadro 1 retrata a definição da hanseníase pelos participantes, que apesar de correta, foi definida de forma incompleta pela maioria, não contemplando todos os aspectos patológicos e fisiológicos da doença, que por conseguinte, demonstra-nos uma falha no processo de saúde-educação que afeta diretamente os pacientes hansênicos que precisam de um acompanhamento de qualidade. 
Quadro 1 - Definição da Hanseníase segundo as falas dos entrevistados.

Uma doença contagiosa e infecciosa, com sintomas como mancha na pele, sensação de formigamento, incapacidade física e dormência nas extremidades. Doença infecciosa crônica, curável. Enfermidade infectocontagiosa causada pelo bacilo de Hansen (Mycobacterium leprae) que causa transtornos dermatológicos, hematológico, neurológicos dentre outros e inclusive invalidez permanente se não tratado a tempo. Costuma infectar muitas pessoas, mas poucas desenvolvem a doença, afetando nervos, principalmente de mãos, pés e olhos. Esta, por sua vez, pode passar em torno de 5 anos para gerar manifestações. Tem um período de incubação e atualmente está sendo mais rápido. Contaminação através das vias respiratórias ao falar e ao tossir.

Fonte: Autores (2021).

De acordo com a literatura científica a hanseníase é caracterizada como uma doença infectocontagiosa, causada pelo microrganismo Mycobacterium leprae, que atinge principalmente a pele e os nervos periféricos e que, sem intervenções, gera incapacidades, principalmente na região dos olhos, das mãos e dos pés (Da Silva et al, 2014).

A característica principal da hanseníase, é o comprometimento dos nervos periféricos que tem um enorme potencial para o desenvolvimento de incapacidades físicas que podem evoluir para deformidades. Dentre as principais manifestações, destacam-se: a perda ou diminuição de sensibilidade, as atrofias, paresias e paralisias musculares (Batista, Vieira, \& De Paula, 2014).

Se não for realizado o tratamento no período inicial, a doença quase sempre evolui, torna-se transmissível e pode atingir pessoas de qualquer sexo ou idade, inclusive crianças e idosos. Essa evolução ocorre de forma lenta e progressiva, podendo levar a incapacidades físicas. A bactéria é transmitida pelas vias respiratórias por meio de contato próximo e prolongado com um doente com hanseníase que não está sendo tratado (Brasil, 2017).

O Quadro 2 apresenta a fala comum dos participantes, referenciando seu conhecimento sobre as principais sequelas ocasionadas pela Hanseníase.

Quadro 2 - Principais sequelas ocasionadas pela Hanseníase.

Lesão dos nervos periféricos. Diminuição da força, sensação de choque, pele seca, falta de suor e dor. Perca de forca que impõe limitação física para usar mãos ou andar. Hematológicas, neurológicas, motoras... Variam de situações mais simples, como diminuição ou perda da força muscular, sensibilidade tátil e dolorosa, mas podem comprometer de forma mais intensa os nervos e causar malformações e dores em mãos, pés, olhos e áreas circunvizinhas. A perda da sensibilidade pode levar a perda de membros e cortes. Dificuldade para andar por causa da dormência. Perda de sensibilidade no local das manchas, perda de força com limitações física em casos mais graves. Sua maior morbidade associa-se aos estados reacionais e ao acometimento neural que podem causar incapacidades físicas e deformidades permanentes. Mãos em garra, perda da força, úlceras na córnea.

Fonte: Autores (2021).

Como observa-se no Quadro 2, o conhecimento referente as sequelas ocasionadas pela hanseníase destes profissionais de saúde apresentam limitações, uma vez que não foram relatadas sobre as sequelas em face, como cegueira, perfuração do septo, ferimentos na região interna do nariz, ectrópio, madarose, triquíase, entre outras. Por conseguinte, esse déficit na compreensão da patologia, faz com que o atendimento a estes pacientes não se torne totalmente abrangente.

Segundo Duarte e colaboradores (2014), as principais deformidades e incapacidades ocasionadas pela hanseníase podem ser devido a fatores neurogênicos e inflamatórios. Dentre as causas neurogênicas pode haver uma perda sensitiva, motora e autonômica, sendo as complicações mais comuns o lagoftalmo parcial ou total, triquíase, opacidade da córnea, ausência de sensibilidade da córnea, madarose, garras rígidas ou móveis, ressecamento de pele, hipotrofias, úlcera e reabsorção óssea. Já em relação às causas inflamatórias, encontram-se as reações hansênicas.

O Quadro 3 apresenta a fala comum dos participantes mostrando a percepção dos mesmos em relação ao impacto ocasionado pelas sequelas hansênicas, na vida das pessoas atingidas pela Hanseníase. 
Quadro 3 - Impacto das sequelas hansênicas na vida dos pacientes.

Muitos hansenianos têm sentimentos de vergonha e medo de expor o corpo, e, com tudo isso eles têm dificuldades de aceitação. Altera a qualidade de vida dos portadores. Medo e rejeição das pessoas. Quando surgem problemas leves, os pacientes tendem a seguir sua rotina, porém quando são intensas, podem afetar o trabalho, a realização de suas atividades diárias, baixar autoestima e gerar ansiedade. Necessitando que o profissional busque entendê-lo de forma holística. Deixa a pessoa com depressão as vezes se sente rejeitada. Limitações e lesões comprometem significativamente a qualidade de vida dos pacientes, com auto-estigmatização e vergonha.

Fonte: Autores (2021).

De acordo com o que foi relatado pelos participantes (Quadro 3), as sequelas ocasionam um significante impacto na vida de todos os pacientes atingidos pela hanseníase, podendo haver redução da qualidade de vida dessas pessoas com manifestação de diferentes sentimentos, como medo, vergonha, ansiedade, baixa autoestima, que por sua vez, levam a uma grande limitação de convívio social.

Desta forma, somado as repercussões físicas pode ser ocasionado um sofrimento psíquico que refle no convívio social, no desempenho do trabalho e nas atividades de vida diárias (Gaudenci et al, 2015). Estes fatores promovem uma repercussão no autocuidado, refletida em medo, tristeza, sensação de impotência, ansiedade, baixa autoestima e isolamento social, assim ocasionado em uma evolução negativa da doença (Corrêa et al, 2014).

Como foi observado no perfil dos profissionais da saúde que participaram desta pesquisa, nota-se a ausência de profissionais de psicologia para realizar o acompanhamento e suporte psicológico e de um fisioterapeuta, que é indispensável nas prevenções de incapacidades e no acompanhamento de todo o tratamento.

Grandes são os desafios para reduzir o impacto causado pela hanseníase relacionadas à qualidade de vida, índices de depressão e as incapacidades físicas. Dessa forma, a prevenção de incapacidades em hanseníase inclui um conjunto de medidas a fim de evitar a ocorrência de danos físicos, emocionais e socioeconômicos, sendo a principal forma o diagnóstico precoce (Brasil, 2017).

No entanto, torna-se necessário discutir acerca da prevenção de incapacidades durante a formação e capacitação dos profissionais de saúde, além de incentivar a promoção de ações com propósito de inserir as práticas de autocuidado no cotidiano dos serviços de saúde que atuam no cuidado e orientação das pessoas afetadas pela doença (Lima et al, 2018).

No Quadro 4, destaca-se a fala comum dos participantes relacionando as principais técnicas de autocuidado orientadas aos pacientes com Hanseníase.

Quadro 4 - Técnicas de autocuidado orientadas aos pacientes.

Uso de hidratantes corporal, protetor solar e prevenção a exposição solar durante o tratamento. Observação de aparecimento de lesões em face, pés e mãos. Investigar as competências dos indivíduos com hanseníase atendido na UBS. Considero-me falha nessas orientações, pois meus conhecimentos são da provenientes da época da universidade e algumas leituras extras e dentre as poucas que faço, busco fazer com que o paciente entenda o que é a doença e que pode ter como complicação; que toma a medicação de forma correta, pois o tratamento é lento; ter cuidado ao toque com objetos muito quentes ou gelados e cuidados de higiene com mãos e pés. Ter o cuidado de não andar descalço para não se cortar. Cuidado com a panela quente e ao cortar alimento porque as vezes se corta e não sente. Hábito de higiene mais rigorosos, hidratação de pele e mucosas e no caso dos pés, usar sapatos adequados, uma alimentação cabível no padrão da família e exercícios físicos. Consiste em procedimentos, técnicas e exercícios que ajudam a prevenir incapacidades ou evitar seu agravamento e podem ser realizados em casa ou no trabalho. Exercícios para fortalecimento dos Membros inferiores e superiores.

Fonte: Autores (2021).

Como exposto no Quadro 4, os participantes relataram que existe uma dificuldade nas orientações das práticas de autocuidado, pois os mesmos dispõem apenas de conhecimentos adquiridos durante a universidade, uma vez que, as 
atualizações do processo de educação em saúde pelas gestões tornam-se falhas, em razão de que a maioria dos profissionais não conhecem a patologia de forma abrangente durante a graduação ou até mesmo não tiveram a oportunidade de vivenciarem a prática clínica com a hanseníase.

Apesar da deficiência no processo de educação permanente, os profissionais relataram práticas de autocuidado de extrema importância como a hidratação corporal, prevenção a exposição solar, hábitos de higiene, cuidado com o manuseio de objetos cortantes e de temperaturas elevadas ou muito baixas, além da execução de exercícios físicos e realização de uma abordagem sobre a patologia para o paciente.

Pinheiro e colaboradores (2014) afirmam, que o autocuidado, tem como finalidade promover a educação em saúde para os próprios indivíduos acometidos que podem cuidar de si próprios, visto que as capacidades necessárias para esta prática se resumem nas habilidades que foram desenvolvidas ao longo da vida, principalmente na presença de problemas de saúde.

Corroborando com os achados, Lima e colaboradores (2018) afirmam que é de suma importância orientar os pacientes durante as consultas acerca da necessidade de hidratação e lubrificação de mãos e pés com o uso de hidratantes corporais e imersão de membros em recipientes com água em temperatura adequada, por 10 minutos. A hidratação e lubrificação da pele são usadas em pele seca e hiperqueratósica, a fim de melhorar as condições da pele, preparando-a para os exercícios indicados à prevenção de incapacidades. Outras orientações necessárias se referem à utilização diária de acessórios, bem como realizar um acompanhamento oftalmológico.

O Quadro 5 apresenta a fala comum dos participantes, referenciando seu conhecimento sobre os tópicos utilizados na Avaliação Neurológica Simplificada (ANS).

Quadro 5 - Principais pontos abordados na "Avaliação Neurológica Simplificada.

Avaliação da face, olhos, palpação dos nervos, avaliação da força e classificação do grau de incapacidade. Anamnese, nível de consciência, reflexo equilíbrio e sensibilidade. Avalia sensação tátil, térmica e dolorosa do paciente, nos primeiros pontos que costumas acometer os nervos que causam malformações nas mãos, pés e olhos. Para isso, faz-se uso de monofilamentos, "alfinetes", gelo e algum material quente. Avaliação dermatológica identificação de lesões de pele com alteração de sensibilidade; avaliação neurológica - identificação de neurites, incapacidades e deformidades..." não conheço".

Fonte: Autores (2021).

No Quadro 5, os participantes relataram que uma das formas de avaliação abordada na ANS é a utilização do gelo ou algum material quente para realização da avaliação da sensibilidade. Apesar de ser um dos recursos utilizados para avaliação da sensibilidade de uma forma geral em pacientes neurológicos, não se apresenta como um método incluso na ANS, utilizada de forma direcionada apenas em pacientes com.

De acordo com Lehman (1997), a avaliação de sensibilidade deve ser realizada através do uso de estesiômetro ou caneta esferográfica comum, além disso, afirma que os principais pontos abordados são: a história, ocupação, queixas do paciente, inspeção, palpação dos nervos, teste de força muscular e de sensibilidade.

É importante ressaltar que foram apresentados os pontos gerais da ANS, mas não de forma detalhada sobre o que realmente é avaliado, além disso, um dos participantes relatou que não tinha conhecimento sobre a avaliação neurológica simplificada, portanto, não utilizava. Fato que pode ser evidenciado com os dados quantitativos já apresentados, onde 27,27\% dos participantes conhecem, mas nunca utilizaram e 18,18\% não conhecem.

A aplicação da Avaliação Neurológica Simplificada deve ser realizada, segundo Lastória e De Abreu (2012), no início, durante e no final do tratamento, com orientações de autocuidados e exercícios tendo como foco as regiões mais acometidas, em especial as áreas dos olhos, nariz, mãos e pés. Para incapacidades indicam-se cirurgias e reabilitação. 
No quadro 6, observamos a fala comum dos participantes, referenciando a percepção dos mesmos sobre a importância do profissional da Atenção primária no acompanhamento do paciente com Hanseníase.

Quadro 6 - Importância do Profissional da Atenção Primária (AP) no acompanhamento do paciente com hanseníase.

Primordial, embora sendo uma doença muito antiga poucas pessoas conhecem, e a atenção primária é quem trata a hanseníase. Somos a porta de entrada para prevenção, promoção, diagnóstico e tratamento da doença. Além de gerenciar as atividades de controle e sistemas de registro de consulta. É de extrema importância, pois é o profissional que está mais próximo do paciente, que entende sua realidade de vida e suas influências culturais, facilitando compreender o surgimento e estratégias para adesão efetiva do mesmo. Porém, sinto falta de atualização e aperfeiçoamentos nessa área. $O$ profissional que atua na APS deve trabalhar com diversas temáticas, devemos buscar conhecimentos, mas só por nós mesmos, às vezes, não há tempo suficiente para contemplar estudos de todas as abordagens. As gestões poderiam estar ajudando nessa busca por atualizações, até para melhora do acompanhamento, fluxo e diminuição dos custos com pacientes acometidos por hanseníase, por exemplo. É de grande importância que é através dele que é identificado os casos. E a prevenção e o tratamento precoce das incapacidades leva a um melhor controle da doença e melhor qualidade de vida sem sequelas ao usuário. É importante para o desenvolvimento de uma vida "normal", além de levar segurança e conforto para o paciente e familiar.

Fonte: Autores (2021).

Observa-se que todos os participantes reconhecem a importância e necessidade do profissional de atenção primária no acompanhamento de pacientes com hanseníase e no processo de prevenção e promoção da saúde.

Corroborando com os achados, Sousa, Da Silva e Xavier (2017), afirmam que a presença de uma equipe interprofissional é de extrema importância na assistência da hanseníase, tendo em vista a necessidade da prestação de cuidados de forma integral, gerando uma maior autonomia no processo de trabalho e assistência da atenção primária.

Um dos fundamentos da atenção primária à saúde é o trabalho interdisciplinar, que tem a Estratégia de Saúde da Família (ESF) como fator prioritário na sua organização. Nessa perspectiva, a ESF tem como apoio os Núcleos de Apoio à Saúde da Família (NASF) a fim de promover a integralidade e auxiliar no desenvolvimento da interdisciplinaridade (Previato \& Baldissera, 2018).

Outro ponto a se destacar, é que apesar da dificuldade de educação sobre hanseníase no sistema de saúde pública, como apresentado no quadro 6 em alguns momentos de fala dos entrevistados, o conhecimento dos profissionais da saúde contempla as principais características da doença, mesmo que de forma incompleta pela maioria dos participantes, entretanto, demonstra-se que possuem algum conhecimento sobre a patologia.

É imprescindível que as atividades educativas realizadas por profissionais de saúde estejam inclusas na rotina dos serviços, principalmente em municípios endêmicos, bem como de divulgar informações e proporcionar o conhecimento da população sobre a hanseníase. Dessa forma, a presença de uma equipe interdisciplinar capacitada e comprometida com a hanseníase influencia diretamente no atendimento de qualidade pela atenção primária (Lana et al, 2014).

\section{Considerações Finais}

O estudo possibilitou uma compreensão acerca do nível de conhecimento dos profissionais de saúde no ambiente de sistema público de saúde, em específico nos programas de saúde da família da zona urbana cidade de Icó-CE, que demonstrou uma escassez desse conhecimento de forma aprofundada sobre a patologia e todo o processo de acompanhamento e orientações, além da necessidade de uma equipe multiprofissional no atendimento a estes pacientes.

Reconhecer estas limitações e ter estratégias para transformá-las a favor de uma formação contínua de educação em saúde e atualizações entre os membros da equipe interprofissional são desafios para que se torne possível fazer avanços acerca das práticas do autocuidado e autonomia do paciente hansênico em relação ao tratamento e à doença. 
Faz-se necessária a realização de estudos semelhantes em outros programas de saúde da família (PSF) e em centros de referência que acompanham pacientes com hanseníase, uma vez que ainda existem poucas pesquisas no Brasil relacionadas ao conhecimento dos profissionais da saúde sobre hanseníase e para que se possa gerar um aprofundamento sobre o conhecimento acerca do perfil desses profissionais em diferentes regiões do país.

Acredita-se que uma das principais contribuições do estudo seja fornecer um referencial norteador para melhoria de serviços especializados no atendimento de hanseníase, podendo proporcionar uma sensibilização dos profissionais sobre a importância de se capacitar, conhecer e orientar a prática do autocuidado para o público alvo em questão, permitindo o desenvolvimento de uma assistência mais humanizada baseada na educação em saúde, sendo de fundamental importância para a prevenção de incapacidades geradas pela doença.

Por fim, observa-se a necessidade de desenvolver estudos que busquem demonstrar a importância do conhecimento dos profissionais de saúde acerca do autocuidado na hanseníase de forma prática e eficaz. Ademais, destaca-se a relevância de se verificar de que forma essa escassez de conhecimento sobre práticas de autocuidado na hanseníase impacta na assistência de qualidade para esses pacientes.

\section{Referências}

Batista, T. V. G., Vieira, C. S. C. A. \& De Paula, M. A. B. (2014). A imagem corporal nas ações educativas em autocuidado para pessoas que tiveram hanseníase. Physis Rev de Saúde Colet, 24 (1), 89-104.

Brasil. (2018). Ministério da Saúde. Boletim epidemiológico hanseníase. Fortaleza: Núcleo de Vigilância Epidemiológica. Secretaria da Saúde do Estado do Ceará.

Brasil. (2020). Ministério da Saúde. Boletim epidemiológico hanseníase. Fortaleza: Núcleo de Vigilância Epidemiológica. Secretaria da Saúde do Estado do Ceará.

Brasil. (2019). Ministério da Saúde. Guia de Vigilância em Saúde. Secretaria de Vigilância em Saúde, Coordenação-Geral de Desenvolvimento da Epidemiologia em Serviços. (3a ed.).

Brasil. (2017). Ministério da Saúde. Secretaria de Vigilância em Saúde. Departamento de Vigilância e Doenças Transmissíveis. Guia prático sobre a hanseníase.

Brasil. (2009). Ministério da Saúde. Secretaria de Vigilância em Saúde. Programa Nacional de Controle da Hanseníase. Relatório de gestão maio de 2007 a dezembro de 2008 .

Ceccim, R. B. (2005). Educação Permanente em Saúde: desafio ambicioso e necessário. Interface (Botucatu), 9 (16), $161-77$.

Ceretta, D. R., Rotoli, A., Cargnin, M. C. S. \& Aires, M. (2012). Grupo de educação em saúde como ferramenta de trabalho com agentes comunitários de saúde: prevenção da hanseníase. Revista de Enfermagem, 8 (8), 208-217.

Corrêa, B. J., Marciano, L. H. S. C., Nardi, S. T., Marques, T., De Assis, T. F. \& Prado, R. B. R. (2014). Associação entre sintomas depressivos, trabalho e grau de incapacidade na hanseníase. Acta Fisiatr, 21 (1), 1-5.

D’Azevedo, S. S. P., De Freitas, E. M., Do Nascimento, L. O., Santos, D. C. M. \& Do Nascimento, R. D. (2018). Percepção de pacientes com hanseníase acerca dos grupos de autocuidado. Rev enferm UFPE on line, 12 (6), 1633-9.

Da Silva, J. S. R., Palmeira, I. P., Sá, A. M. M., Nogueira, L. M. V. \& Ferreira, A. M. R. (2014). Fatores sociodemográficos associados ao grau de incapacidade física na hanseníase. Rev Cuid, 9 (3), 2338-48.

Duarte, L. M. C. P. S., Simpson, C. A., Silva, T. M. S., Moura, I. B. L. \& Isoldi, D. M. R. (2014). Ações de autocuidado de pessoas com hanseníase. Rev enferm, 8 (8), 2816-22.

Ferreira, L., Barbosa, J. S. A., Esposti, C. D. D. \& Da Cruz, M. M. (2019). Educação Permanente em Saúde na atenção primária: uma revisão integrativa da literatura. Saúde Debate, 43 (120), 223-239.

Galan, N. G. A., Beluci, M. L., Marciano, L. H. S. C., Ruiz, R. B. P., Oliveira, N. G. G., Bonini, A. G., Arakaki, F. R. \& Guimarães, G. S. (2016). Avaliação da prática do autocuidado domiciliar em hanseníase. Hansen Int, 41 (1-2), 37-45.

Gaudenci, E. M., Nardelli, G. G., Neto Almeida, O. P., Malaquias, B. S. S., Carvalho, B. T. \& Pedrosa, L. A. K. (2015). Qualidade de vida, sintomas depressivos e incapacidade física de pacientes com hanseníase. Hansen Int, 40 (2), 48-58.

Gil, A. C. (2014). Métodos e técnicas de pesquisa social. (6a ed.). Atlas Editora S.A.

Lana, F. C. F., Lanza, F. M., Carvalho, A. P. M. \& Tavares, A. P. N. (2014). O estigma em hanseníase e sua relação com as ações de controle. Rev Enferm UFSM, 4 (3), 556-565. 
Research, Society and Development, v. 10, n. 13, e556101321427, 2021

(CC BY 4.0) | ISSN 2525-3409 | DOI: http://dx.doi.org/10.33448/rsd-v10i13.21427

Lastória, J. C. \& De Abreu, M. A. M. M. (2012). Hanseníase: diagnóstico e tratamento. Diagn Tratamento, 17 (4), 173-9.

Lehman, L. F., Orsini, M. B. P., Fuzikawa, P. L., Lima, R. C. \& Gonçalves, S. D. (1997). Avaliação neurológica simplificada. Belo Horizonte: ALM Internacional.

Lima, M. C. V., Barbosa, F. R., Santos, D. C. M., Do Nascimento, R. D. \& D’Azevedo, S. S. P. (2018). Práticas de autocuidado em hanseníase: face, mãos e pés. Rev Gaúcha Enferm, 39.

Ludwig, A. C. W. (2012). Fundamentos e prática de Metodologia Científica. (2a ed.). Vozes Editora.

Marconi, M. A. \& Lakatos, E. M. (2010). Fundamentos de metodologia científica. (7a ed.). Atlas Editora S.A.

Morais, J. R \& Furtado, E. Z. L. (2018). Grau de incapacidade física de pacientes com hanseníase. Rev enferm UFPE on line, 12 (6), 1625-32.

Moura, E. G. S., Araújo, A. P. M., Da Silva, M. C. R., Cardoso, B. A., Holanda, M. C. S., Conceição, A. O. \& Dias, G. A. S. (2017). Relação entre a classificação Internacional de Funcionalidade, Incapacidade e Saúde (CIF) e a limitação de atividades e restrição à participação de indivíduos com Hanseníase. Cad. Saúde Colet, 25 (3), 355-361.

Oliveira, S. B., Ribeiro, M. D. A., Silva, J. C. A. \& Silva, L. N. (2017). Avaliação do nível de informação sobre hanseníase de profissionais da estratégia saúde da família. Rev Pesq Saúde, 18 (3), 139-143.

Pinheiro, M. G. C., Silva, S. Y. B., Silva, F. S., Ataide, C. A. V., De Lima, I. B. \& Simpson, C. A. (2014). Conhecimento sobre prevenção de incapacidades em um grupo de autocuidado em hanseníase. Revista Mineira de Enfermagem, 18 (4), 895-906.

Previato, G. F. \& Baldissera, V. D. A. (2018). Retratos da prática interprofissional colaborativa nas equipes da atenção primária à saúde. Rev. Gaúcha Enferm, 39.

Rodrigues, F. F., Calou, C. G. P., Leandro, T. A., Antezana, F. J., Pinheiro, A. K. B., Silva, B. P. \& Alves, M. D. S. (2015). Conhecimento e prática dos enfermeiros sobre hanseníase: ações de controle e eliminação. Rev Bras Enferm, 68 (2), 297-304.

Sousa, G. S., Da Silva, R. L. F. \& Xavier, M. B. (2017). Hanseníase e Atenção Primária à Saúde: uma avaliação de estrutura do programa. Saúde Debate, 41 (112), 230-242.

Souza, I. A., Ayres, J. A., Meneguin, S. \& Spagnolo, R. S. (2014). Autocuidado na percepção de pessoas com hanseníase sob a ótica da complexidade. Esc. Anna Nery, 18 (3), 510-514. 